\title{
Estudio Cualitativo de la Práctica Fonoaudiológica en el Contexto de Rehabilitación Basada en la Comunidad, en la Quinta Región de Valparaíso
}

\author{
COMMUNITY BASED SPEECH AND LANGUAGE REHABILITATION QUALITATIVE STUDY, IN THE FIFTH REGION \\ OF VALPARAÍSO
}

Angélica Silva R ${ }^{1}$, Gissela Rojas N ${ }^{1}$, Katherine Vásquez $\mathrm{G}^{1}$, Manuel del Campo $\mathrm{R}^{1}$

1. Universidad Santo Tomás. Facultad de Salud, Escuela de Fonoaudiología. Viña del Mar.

\begin{abstract}
RESUMEN
El enfoque de Rehabilitación Basada en la Comunidad (RBC) representa una estrategia cuyo propósito es lograr la plena integración e inclusión de las personas en situación de discapacidad (PsSD) a nivel social. Considerando que la comunicación y el lenguaje humano representan herramientas de expresión del pensamiento, la Asociación Americana de Habla, Lenguaje y Audición (ASHA) insta a promover el desarrollo de una comunicación efectiva como un derecho humano accesible para todas las personas, en consideración de su dignidad inherente. Objetivo: conocer el quehacer de fonoaudiólogos(as) que se desempeñan en el contexto de RBC, en la Quinta Región de Valparaíso. Método: investigación de tipo cualitativa, con enfoque fenomenológico. Se realizó una entrevista semiestructurada y posteriormente se procedió a realizar un análisis de contenido. Resultados: Los fonoaudiólogos(as) ejecutan acciones atingentes a los cinco pilares de la RBC y se observa la práctica de actos vinculados a la gestión del intersector. Conclusiones: Los profesionales adoptan la estrategia RBC incorporada en la visión de la institución en la que se encuentran insertos, adoptando un perfil orientado a la transdisciplina.
\end{abstract}

(Silva A, Rojas G, Vásquez K, Del Campo M, 2018. Estudio Cualitativo de la Práctica Fonoaudiológica en el Contexto de Rehabilitación Basada en la Comunidad, en la Quinta Región de Valparaíso. Cienc Trab. Sep-Dic; 20 [63]: 126-130).

Palabras clave: FONOAUDIOLOGÍA, REHABILITACIÓN, ORGANIZACIONES NO GUBERNAMENTALES.

\section{ABSTRACT}

The Community Based Rehabilitation (CBR) approach represents a strategy whose purpose is to achieve full social integration and inclusion of people with disabilities. Considering that communication and human language represent tools of expression of thought, the American Speech, Language and Hearing Association (ASHA) advocates the development of effective communication promotion, as a human right accessible to all people, considering its inherent dignity. Objective: to analyze speech and language pathologist's CBR practices in the fifth region of Valparaiso. Method: qualitative research, with a phenomenological approach. A semi-structured interview was conducted followed by a content analysis. Results: speech and Language pathologists carry out actions related to the five CBR key components and also the practice of actions in accord to intersector. Conclusions: The professionals adopt the CBR approach incorporating the institutional vision of service in which they are inserted and adopting a profile oriented to transdisciplinary.

Key words: SPEECH, LANGUAGE AND HEARING SCIENCES, REHABILITATION, NON-GOVERNMENTAL ORGANIZATIONS.

\section{INTRODUCCIÓN}

El enfoque de Rehabilitación Basada en la Comunidad (RBC) representa una estrategia cuyo propósito es lograr la plena integración e inclusión de las personas en situación de discapacidad (PsSD) a nivel

Correspondencia / Correspondence:

Sra. Angélica Silva Ríos

Av. Limonares 190, Viña del Mar, Valparaíso, Chile.

Tel.: +56 322443019

e-mail: asilvar@santotomas.cl

Código Postal: 2561780

Recibido: 01 de marzo de2018 / Aceptado: 30 de julio de 2018 social, con el fin último de que los individuos puedan alcanzar la independencia y el desarrollo máximo de su potencial. ${ }^{1}$

En Chile, la iniciativa de la RBC inicia una evolución incipiente gracias a la promulgación de la Ley 19.284, la cual establece normas para la plena integración social de personas con discapacidad. ${ }^{2}$ No es hasta el año 2003 cuando se incorpora como lineamiento de la política pública la terminología RBC propiamente tal en el país, estableciéndose que en este esfuerzo deben participar: la ciudadanía, organizaciones de personas en situación de discapacidad (OPD), sus familias y organizaciones no gubernamentales (ONGs). ${ }^{3}$ En el año 2010, en respuesta a la Convención de las Naciones Unidas sobre los derechos de las personas con discapacidad ${ }^{4}$, en Chile se promulga la Ley $20.422^{5}$, que establece normas sobre igualdad de oportunidades e inclusión social de personas con discapacidad, cuyo objetivo es "asegurar el derecho a la igualdad de oportunidades de las personas 
con discapacidad, con el fin de obtener su plena inclusión social, asegurando el disfrute de sus derechos y eliminando cualquier forma de discriminación fundada en la discapacidad". ${ }^{5}$

Durante el año 2012, la Organización Mundial de la Salud (OMS) realiza una actualización a los manuales existentes de RBC, publicando cinco guías correspondientes a los pilares de la estrategia, a saber: salud, educación, trabajo, participación social y fortalecimiento. ${ }^{6}$

Considerando que la comunicación y el lenguaje humano representan herramientas de expresión del pensamiento ${ }^{7}$, la Asociación Americana de Habla, Lenguaje y Audición (ASHA) insta a promover el desarrollo de una comunicación efectiva como un derecho humano accesible para todas las personas, en consideración de su dignidad inherente. ${ }^{8}$

En este contexto, cabe mencionar que la fonoaudiología es la disciplina encargada de la evaluación, diagnóstico, rehabilitación, promoción de la salud y prevención de los trastornos del lenguaje, habla, deglución, audición, voz y comunicación. ${ }^{9}$

Desde el punto de vista de la convivencia de todos y todas en un contexto comunitario, los fonoaudiólogos(as) tienen una obligación fundamental expresada en su rol, la que cruza un amplio espectro de actividades que van desde la promoción de la salud hasta la rehabilitación de trastornos que afectan la comunicación, la deglución y calidad de vida de PsSD. ${ }^{10}$

Con estos antecedentes en mente, el objetivo de la presente investigación es conocer el quehacer de fonoaudiólogos(as) que se desempeñan en el contexto de RBC, en la Quinta Región de Valparaíso.

\section{Material y métodos}

Esta investigación es de tipo cualitativa ${ }^{11}$, con un enfoque fenomenológico. ${ }^{12}$ Se realizó una entrevista semiestructurada y, posteriormente, se procedió a realizar un análisis de contenido, identificando categorías de significado relevantes a partir de los datos recopilados.

El universo de interés fue fonoaudiólogos(as), chilenos o extranjeros que se desempeñaran en el contexto de RBC, con al menos un año de experiencia en el sector, en la región de Valparaíso.

La técnica de muestreo utilizada fue "snow ball"13, ya que tras identificar a un(a) fonoaudiólogo(a) en el ámbito antes detallado, se procedió a contactar a otros colegas de su red.

El estudio fue aprobado por el comité de ética científico de la macro zona norte, de la Universidad Santo Tomás, con código 30.16 , e incluye dentro de su desarrollo la firma de un consentimiento informado por parte de los participantes. La investigación suscribe el acuerdo de Helsinski y no supuso la aplicación de métodos que pudieran causar dolor o incomodidad a los participantes.

\section{RESULTADOS}

El quehacer fonoaudiológico fue explorado a través de una entrevista semiestructurada, de la cual emergieron cuatro familias o categorías de significado, como sigue: a) conocimiento, b) acciones, c) gestión del intersector, d) fortaleza y debilidades. Estas familias de significado se proceden a detallar a continuación.

\section{a) Conocimiento de la estrategia RBC}

Se entiende como conocimiento toda la información que maneja el fonoaudiólogo(a) en cuanto al concepto de RBC6 y el rol que este cumple en dicho ámbito.

Ante el reactivo, “¿conoce usted la estrategia de la RBC y su relación con la fonoaudiología?, se observa que los fonoaudiólogos llegan al conocimiento de la estrategia a partir de la visión de las instituciones en las que se insertan y de las que adoptan el modelo.

"Como somos fonoaudiólogos los que creamos esto, cuando abrimos el tema del contenido, de cómo se tenía que desarrollar la institución, nosotros detectamos en qué áreas de la matriz podíamos desarrollarnos de acuerdo a nuestras competencias [...]. Ahora esta institución en particular es súper amplia en cuanto a su visión, como que nosotros cubrimos todas las áreas de la RBC".

(Flgo. $N^{\circ}$ 4, 16 de mayo del 2017)

¿Qué perfil creen debe tener el fonoaudiólogo que trabaje en RBC?; la mayoría de los/as entrevistados/as concordaron en que el fonoaudiólogo/a debe ser proactivo, dispuesto a trabajar en equipo, desligarse del modelo biomédico, así como también de su disciplina, aprendiendo de otros profesionales y especialidades. Algunas citas relevantes al tema son:

"El perfil de un profesional que trabaja en RBC tiene que ampliarse a la transdisciplina más que a la súper especialización de un área”.

(Flga. № 1, 24 de abril del 2017)

"Debe poseer la capacidad de poder integrar distintas miradas, la capacidad de poder desaprender el modelo biomédico; insertarnos en la comunidad, eso creo que es muy relevante [...]".

(Flga. $\mathrm{N}^{\circ}$ 5, 26 de mayo del 2017)

En cuanto a la pregunta, ¿en qué ámbitos de la RBC puede desempeñarse el fonoaudiólogo(a)?, los profesionales entrevistados consideran que el fonoaudiólogo tiene las capacidades de insertarse en todos los ámbitos propuestos por la RBC.

"Yo creo que en todas, o sea, jen todas! De hecho, a mí una de las que más me gusta es la de fortalecimiento; en la de fortalecimiento de organizaciones, agrupar, educar a las organizaciones, levantar necesidades en ellas y luego responder, hacer diagnósticos (...)".

\section{b) Acciones}

(Flga. $N^{\circ} 4,16$ de mayo del 2017)

El objetivo de los pilares propuestos por la RBC es orientar a las instituciones en su quehacer, respaldando a las personas en situación de discapacidad. ${ }^{6}$ Para fines prácticos, los resultados se dividieron en los cinco pilares propuestos por la RBC.

\section{Salud}

En este sector ${ }^{14}$ los profesionales refieren realizar principalmente acciones ligadas a la prevención y promoción en salud fonoaudiológica. Entre sus respuestas destaca:

"Comúnmente se hace el segundo semestre lo que es un taller que va dirigido al adulto mayor sin deterioro cognitivo. Es algo preventivo y ese sí va asociado a la comunidad porque, como no es patológico, cualquiera puede venir y ahí se hace un taller de estimulación cognitiva”.

(Flgo. № 3, 4 de mayo del 2017) "El tema que podría ser de prevención es la detección temprana, la detección de las señales de alerta [...]. El año 2011 se hizo una campaña con el SENADIS, se creó una campaña que se llama 
Creando una cultura del autismo en Chile, que es un seminario que ya hacemos todos los años, que ya se repite cada año [...]; eso podría ser como promoción y prevención".

(Flga. $\mathrm{N}^{\circ} 2,25$ de abril del 2017)

"Nosotros, particularmente, trabajamos con adultos, mucho adulto mayor y con esos programas funcionamos con el EMPAM (Examen de medicina preventiva del adulto mayor), hacemos seguimiento; está bien ligado al examen de funcionalidad [...]”.

(Flga. № 6, 07 de junio del 2017)

\section{Educación}

Respecto a los vínculos existentes entre el sistema educativo ${ }^{15}$ y el lugar de atención de los profesionales fonoaudiólogos(as), se menciona que existe una relación estrecha de trabajo colaborativo respecto a los usuarios de las ONGs; es así como se observan relatos como:

"Sí existen, porque vamos a los colegios, universidades, jardines infantiles en todos los que están nuestros niños".

(Flga. № 2, 25 de abril del 2017) "En lo que respecta a planes de intervención, han sido en conjunto con la escuela, realizamos reuniones semestrales con la directora y la educadora diferencial y le damos un plan semestral [...]; vamos constantemente retroalimentándonos por vía telefónica [...]".

(Flga. № 5, 26 de mayo del 2017)

\section{Subsistencia}

Respecto a este pilar, las preguntas se centraron en la participación del fonoaudiólogo en la inserción laboral de las personas en situación de discapacidad. ${ }^{16}$ Esta temática se constata como un tópico relevante para las ONGs, el cual es abordado de acuerdo a la visión institucional en la que se desempeña en el profesional. Existen diversas formas para dar respuesta a este pilar de la RBC como, por ejemplo, desde el acompañamiento de los usuarios en su proceso de ingreso al mundo laboral hasta la tramitación de pensiones de invalidez.

"Eso no es algo que hago solo yo (inserción laboral), sino que es como el norte de la institución [...]; eres como el coach de los que quieren entrar a trabajar y tú los acompañas en el proceso y les prestas los apoyos necesarios desde el principio hasta el final. Puede ser fonoaudiólogo de base, puede ser terapeuta de base, puedes ser psicopedagogo de base, psicólogo, ...no hay distinción profesional".

(Flga. No 1, 24 de abril del 2017)

Otro profesional, por su parte, refiere que:

"No hay inserción laboral, sin embargo, sí hay orientación por lo menos de mi parte, por una cosa de manejo y de conocimiento para todo lo que es la tramitación de pensión de invalidez".

(Flgo. No 3, 04 de mayo, 2017)

\section{Componente social}

Se pudo apreciar que en relación al fomento de la participación plena de las PsSD en la comunidad donde se desenvuelven ${ }^{17}$ todos los fonoaudiólogos lo consideran un punto fundamental en su intervención.

"Sí, de hecho, ese es el fin último. Nosotros siempre decimos que no es la meta que la persona adulta con discapacidad se emplee; es recién el inicio, porque cuando uno es grande y quieres tener tu vida de manera independiente, hacer las cosas que te gusta, consigues trabajo, no es ahí que se terminó tu vida si no que el trabajo lo consigues para poder tener recursos, para tener amigos, para poder salir, para poder crecer".

(Flga. № 1, 24 de abril del 2017) "Sí, nosotros trabajamos con la comunidad; como te digo, tratamos de que todas las persona participen de la comunidad, algunos de ellos van al gimnasio, a otros les gusta ir a restaurantes y ahora el viernes nos vamos a juntar (...)".

(Flga. № 2, 25 de abril del 2017)

\section{Fortalecimiento}

Este pilar cobra relevancia en la inclusión de las PsSD, sus familias y la comunidad; promoviendo la autonomía en los distintos ámbitos de participación con el fin último de reconocer sus derechos inherentes. ${ }^{18}$ Tras realizar un análisis transversal de las respuestas de los entrevistados, destaca la importancia que los fonoaudiólogos(as) le dan a los derechos de las PsSD; además del contacto que generan algunos profesionales con otras entidades de PsSD, creando así un fuerte vínculo que sustenta las acciones y la gestión a nivel del intersector. Algunas respuestas relevantes son:

"El enfoque de derechos en discapacidad es esencial, que se vea a la PsSD como alguien cualquiera, digamos que tiene derecho a tomar las riendas de su propia vida y ejercer sus derechos en todos los ámbitos y eso sí es algo que se realiza mucho".

(Flga. № 1, 24 de abril del 2017)

“Tenemos también un grupo de autogestión que se llama TEAPOLIS, ellos ya están desligándose de nosotros, están siendo sus propios gestores y... están creciendo bastante, están teniendo liderazgo y nosotros solamente los guiamos o los orientamos y ellos son los que lideran y también participan de la comunidad [...]".

(Flga. № 2, 25 de abril del 2017)

\section{c) Gestión del Intersector}

Este término se refiere al modo de actuar y el ejercicio de coordinación entre distintas instituciones. Las guías RBC mencionan que el contacto con diversas entidades es primordial a la hora de querer desarrollar la RBC, siendo un componente esencial en ella. ${ }^{6}$ En este aspecto los fonoaudiólogos(as) entrevistados mencionan realizar algunas acciones relativas a la gestión, empero aún responden a una aproximación intuitiva que necesita ser sistematizada.

Algunas citas sobresalientes en este respecto son:

"Es bastante fácil por la cercanía que tienen las autoridades con este centro [...]“.

(Flgo. № 3, 16 de mayo del 2017) "Tenemos harto contacto con dirigentes vecinales que nos ayudan a contactar a ciertas personas [...]. Sí, nosotros sí activamos esto como de los monitores en terreno, del barrio que sugieren las guías de RBC, de cómo incentivar y empoderar a las personas a nivel local [...]".

(Flga. № 4, 16 de mayo del 2016)

\section{Fortalezas y Debilidades}

$\mathrm{Al}$ explorar tópicos relacionados con las necesidades y buenas prácticas advertidas en el contexto de RBC, los entrevistados(as) refieren que el quehacer diario de los profesionales se encuentra en un proceso de maduración, donde deben conversar tanto la teoría como la experiencia para que la prestación de los servicios ofertados pueda efectivamente impactar de manera positiva en la calidad comunicativa de los usuarios. 
Como ejemplo de esto destacamos los siguientes discursos:

"Falta experiencia, principalmente; o sea, yo creo que falta primero hacer más difusión de lo que es RBC [...], quizás existirian más profesionales, que incluso haciendo lo que ya hacen pudieran decir: "oh, en verdad parece que yo también estoy alineada con estos principios, solo que no tenía idea cómo se llamaba ni que existían".

(Flga. No 1, 24 de abril del 2017) "Yo veo que lo que falta en Chile es pasar de un modelo rehabilitador, un modelo más clínico, a un modelo social, que no nos centremos tanto en el déficit o lo que le cuesta a la persona, sino que en sus potencialidades y en sus derechos y planificar desde el enfoque de calidad de vida, centrados en las personas [...]".

(Flga. № 2, 25 de abril del 2017) "Yo creo que no hay mucho fonoaudiólogo en formación para el trabajo de la persona en situación de discapacidad, si bien hay gente que ocupa los modelos de empleo con apoyo y todo eso, pero la formación misma, la capacitación... eso no lo hace y yo creo que lo puede hacer perfectamente [...]".

(Flga. $N^{\circ}$ 4, 16 de mayo del 2017) "Si uno entiende que tú como fonoaudiólogo eres especialista en comunicación, que es algo inmensamente amplio que está en todas partes -si no en todo el mundo- puedes tener una amplitud en tu campo laboral gigante, pero si es que tú mismo te vuelves flexible y lo amplías [...]".

(Flga. № 1, 24 de abril del 2017)

\section{DISCUSIÓN}

Esta investigación buscó explorar el quehacer fonoaudiológico en el contexto de la RBC. Todos los profesionales entrevistados concuerdan que para poder realizar RBC, o estar dispuestos a trabajar con ella, es indispensable que se desarrolle un pensamiento crítico y se entreguen herramientas durante la formación académica del pregrado, debido a que al momento de tener que trabajar bajo las directrices de la estrategia, puede resultar un tanto extraño el no ejecutar actividades ligadas más al modelo biomédico, entiéndase, evaluación, diagnóstico y rehabilitación. ${ }^{9}$
El análisis de la información recopilada permite reflexionar respecto a que los(as) fonoaudiólogos(as) desempeñan labores pertinentes a los pilares de la RBC; sin embargo, estas actividades pueden fortalecerse mediante la instrucción desde el pregrado. Lo anterior demuestra que desde un punto de vista profesional se han hecho adecuaciones para ajustar la práctica al contexto de RBC, independientemente de no contar con herramientas teóricas de base.

El documento "Orientaciones metodológicas para el desarrollo de estrategias de rehabilitación en APS" considera como rehabilitadores a los profesionales kinesiólogos y terapeutas ocupacionales ${ }^{19}$, es decir, orienta la prestación de servicios esencialmente hacia la rehabilitación física, se advierte entonces la oportunidad de fortalecer la salud mental, comunicativa y social de los integrantes de la comunidad, desde la fonoaudiología.

Los fonoaudiólogos en Centros Comunitarios de Rehabilitación (CCR) cumplen el rol de guía para los usuarios y sus familias, integran un equipo interdisciplinario y se instruyen acerca del contexto biopsicosocial en el cual se desenvuelven las personas de la comunidad en la que desarrollan sus labores. Cabe resaltar la necesidad de que los profesionales fonoaudiólogos(as) sean capaces de detectar las barreras que enfrentan los usuarios, para así desarrollar estrategias en que podrían superarlas. ${ }^{20}$

Dentro de los hallazgos principales de la investigación podemos mencionar que los fonoaudiólogos insertos en ONGs se apropian de la estrategia RBC, realizando acciones inclusivas desde un enfoque biopsicosocial, considerando, en mayor o menor medida, los distintos pilares declarados. Este despojarse de la intervención tradicional puede deberse a la mayor autonomía en el ámbito laboral, ya que los profesionales no se encuentran sometidos a metas trazadas por organismos gestores, que por lo general se orientan al número de atenciones realizadas, como es el caso de atención primaria en salud (APS). Del mismo modo, esta mayor flexibilidad en la inserción del fonoaudiólogo a los equipos de trabajo le permite transitar de lo multiprofesional, abordaje en el que los conocimientos se fragmentan en relación a las disciplinas de origen, a lo transdisciplinar, donde se asume la naturaleza plural de los sujetos, trascendiendo a las áreas y permitiendo desarrollar una concepción integradora del conocimiento. 


\section{REFERENCIAS}

1. OIT-UNESCO-OMS. R.B.C.: estrategia para la rehabilitación, la igualdad de oportunidades, la reducción de la pobreza y la integración social de las personas con discapacidad [en línea]. Ginebra: OMS; 2004 [citado ene 2018]. Disponible en: http://apps.who.int/iris/bitstream/10665/43126/1/ 9243592386_spa.pdf

2. Chile. Ministerio de Planificación y Cooperación (MIDEPLAN). LEY-19284; Establece normas para la plena integración social de personas con discapacidad [en línea]. Santiago de Chile: Biblioteca del Congreso Nacional; 1994 [citado 22 ene 2018]. Disponible en: https://www.leychile.cl/Navegar? idNorma $=30651$ ctidParte $=$

3. Ministerio de Salud (MINSAL). Orientaciones para la implementación del Modelo de Atención Integral de salud familiar y comunitaria; dirigido a equipos de salud [en línea]. Santiago de Chile: MINSAL; 2013 [citado ene 2018]. Disponible en: http://buenaspracticasaps.cl/wp-content/uploads/ 2013/11/Orientaciones-para-la-implementacion-del-Modelo-de-Atenci\%C3\%B3n-Integralde-Salud-Familiar-y-Comunitaria_DIVAP_2013.pdf

4. Chile. Ministerio de Relaciones Exteriores. Decreto 201; Promulga la Convención de las Naciones Unidas sobre los derechos de las personas con discapacidad y su protocolo facultativo [en línea]. Santiago de Chile: Ministerio de Realciones Exteriores; 2008 [citado 22 ene 2018]. Disponible en: https://www.leychile.cl/Navegar?idNorma=278018

5. Chile. Ministerio de Planificación y Cooperación (MIDEPLAN). Ley 20422; Establece normas sobre igualdad de oportunidades e inclusión social de personas con discapacidad [en línea]. Santiago de Chile: MIDEPLAN; 2010 [citado 22 ene 2018]. Disponible en: https://www.leychile.cl/Navegar? idLey=20422

6. OMS-UNESCO-OIT-OPS-IDDC. Rehabilitación basada en la comunidad: guias para la RBC; Folleto Introducción [en línea]. Ginebra: OMS; 2012 [citado ene 2018]. Disponible en: http://apps.who.int/iris/bitstream/ 10665/44809/42/9789243548050_Introducci\%C3\%B3n_spa.pdf

7. Everaert $M B H_{1}$, Huybregts $M$, Berwick $R$, Chomsky $N_{1}$ Tattersall I, Moro $A_{\text {, }}$ Bolhuis J. What is Language and How Could it Have Evolved? Trends Cogn Sci. 2017; 21(8):569-571.

8. American Speech-Language-Hearing Association (ASHA). About the American Speech-Language-Hearing Association [on line]. Rockville: ASHA; 2018 [cited 22 ene 2018]. Available from: http://www.asha.org/about/

9. Martínez L, Cabezas $C$, Labra M, Hernández R, Martínez L, Cerutti M, Malebrán C. La logopedia en Iberoamérica. En: ResearchGate [sitio web] XXV Congreso de Logopedia, Foniatría y Audiología; 28-30 jun 2006; Granada, España [citado ene 2018]. Disponible en: https://www.researchgate.net/ publication/228870137_LA_LOGOPEDIA_EN_IBEROAMERICA
10. Vega YE, Torres AM, del Campo MN. Análisis del rol del fonoaudiólogo(a) en el sector salud en Chile. Cienc Trab [en línea] 2017 [citado ene 2018]; 19(59):76-80. Disponible en: https://scielo.conicyt.cl/pdf/cyt/v19n59/07182449-cyt-19-59-00076.pdf

11. Taylor SJ, Bogdan R. Introducción a los métodos cualitativos de investigación. México: Paidós; 1986.

12. Hernández $R$, Batista I. Metodología de la investigación. $6^{a}$ ed. México: McGRAW-Hill Interamericana; 2014.

13. Rodríguez D, Valldeoriola J. Metodología de la Investigación [en línea]. Barcelona: UOC; 2009 [citado ene 2018]. Disponible en: https://es.scribd. com/doc/294987302/Metodologia-de-La-Investigacion-Rodriguez-y-Valldeoriola

14. OMS-UNESCO-OIT-OPS-IDDC. Rehabilitación basada en la comunidad: guias para la RBC. Componente de Salud [en línea]. Ginebra: OMS; 2012 [citado sep 2018]. Disponible en: http://apps.who.int/iris/bitstream/ 10665/44809/3/9789243548050_Salud_spa.pdf

15. OMS-UNESCO-OIT-OPS-IDDC. Rehabilitación basada en la comunidad: guias para la RBC. Componente de Educación [en línea]. Ginebra: OMS: 2012 [citado sep 2018]. Disponible en: http://whqlibdoc.who.int/publications/2012/9789243548050_Educaci\%C3\%B3n_spa.pdf?ua=1

16. OMS-UNESCO-OIT-OPS-IDDC. Rehabilitación basada en la comunidad: guías para la RBC. Componente de Subsistencia [en línea]. Ginebra: OMS; 2012 [citado sep 2018]. Disponible en: http://apps.who.int/iris/bitstream/ 10665/44809/4/9789243548050_Subsistencia_spa.pdf

17. OMS-UNESCO-OIT-OPS-IDDC. Rehabilitación basada en la comunidad: guías para la RBC. Componente Social [en línea]. Ginebra: OMS; 2012 [citado sep 2018] Disponible en: http://apps.who.int/iris/bitstream/ 10665/44809/5/9789243548050_Social_spa.pdf

18. OMS-UNESCO-OIT-OPS-IDDC. Rehabilitación basada en la comunidad: guias para la RBC. Componente de Fortalecimiento [en línea]. Ginebra: OMS; 2012 [citado sep 2018]. Disponible en: http://apps.who.int/iris/bitstream/ 10665/44809/2/9789243548050_Fortalecimiento_spa.pdf

19. MINSAL. Orientaciones metodológicas para el desarrollo de las estrategias de rehabilitación en A.P.S. 2010 -2014 [en linea]. Santiago de Chile: MINSAL; 2010 [citado sep 2018]. Disponible en: www.senadis.gob.cl/ descarga/i/225/documento

20. Tapia S, Espinoza F, Herrera P, Venegas D. Caracterización de fonoaudiólogos/as insertos/as en Centros Comunitarios de Rehabilitación. Rev Chil Fonoaudiol [en línea] 2016 [citado sep 2018]; 15(nov):1-13. Disponible en: https://revfono.uchile.cl/index.php/RCDF/article/view/44186/46192 